Z Herz-Thorax- Gefäßchir 2008 · 22:98-102 DOI 10.1007/s00398-008-0621-9 Online publiziert: 11. März 2008 ๑) Springer Medizin Verlag 2008

\section{T. Attmann}

Klinik für Herz- und Gefäßchirurgie Universitätsklinikum Schleswig-Holstein, Kiel, BRD
„The Kiel Experience"

(RV) und Pulmonalarterien durch Interposition einer nichtklappentragenden Gefäßprothese geschaffen.

Die hieraus bei einer Vielzahl der Patienten resultierende Pulmonalklappeninsuffizienz sah man in der Vergangenheit als ein vernachlässigbares Vitium an. Im Langzeitverlauf wurden jedoch negative Auswirkungen auf die Hämodynamik des rechten und linken Ventrikels beobachtet $[5,6,7]$. Die chronische Volumenbelastung des RV führt zu Dilatation und eingeschränkter systolischer und diastolischer Funktion $[8,9]$. Aus der Dilatation des Trikuspidalklappenannulus resultiert eine neu auftretende oder größer werdende Trikuspidalklappeninsuffizienz. Die Kombination beider Vitien ist für die Dilatation des rechten Herzens verantwortlich. Längerfristig gehen atriale und ventrikuläre Rhythmusstörungen, Herzinsuffizienz und ein erhöhtes Risiko für den plötzlichen Herztod einher. Die genannten Sequelae sind an eine Pulmonalklappeninsuffizienz nach Fallot-Korrektur gekoppelt [10]. Die Verbesserung der RV-Funktion, eine Reduktion der Inzidenz von Arrythmien sowie eine größere körperliche Belastbarkeit konnten durch den Erhalt der Pulmonalklappenfunktion bzw. deren Wiederherstellung zum richtigen Zeitpunkt erreicht werden $[7,11]$.
Dieser „richtige“ Zeitpunkt konnte bislang jedoch nicht eindeutig bestimmt werden. Üblicherweise wird die Indikation zur chirurgischen Therapie einer Pulmonalklappeninsuffizienz gestellt, wenn die Patienten symptomatisch werden, die körperliche Belastbarkeit sinkt, Arrhythmien auftreten, der rechtsventrikuläre Diameter zunimmt und sich die RVFunktion verschlechtert. Zu diesem Zeitpunkt können jedoch bereits irreversible Veränderungen des Myokards eingetreten sein [11].

Häufig erhalten die betroffenen Patienten in der frühen Adoleszenz einen Pulmonalklappenersatz mit einer biologischen Prothese. Hier kommen ein Homograft oder eine klappentragende Rinderhalsvene (Contegra ${ }^{\circledR}$ ) zum Einsatz. Jedoch neigen beide Prothesentypen zu Verkalkung und Degeneration, weshalb meist bereits nach wenigen Jahren erneut Pulmonalklappenstenose oder auch -insuffizienz in Erscheinung treten [12]. Rezidivierende Operationen mit Einsatz der Herz-Lungen-Maschine (HLM) waren bisher unumgänglich.

Der chirurgische Pulmonalklappenersatz ist mit niedriger Morbidität und so gut wie keiner Letalität verbunden. Jedoch bestehen bei diesen äußerst anspruchsvollen, häufig viele Stunden dauernden Reoperationen spezifische Ri- 
siken: Blutverlust mit Substitution durch Fremdblut, Verletzung von Gefäß- und Nervenstrukturen bei vorhandenen Verwachsungen sowie Wundheilungsstörung und Instabilität des Sternums [13]. Darüber hinaus ist im Anschluss an die Operation ein längerer stationärer Aufenthalt erforderlich.

\section{Wie macht man das?}

Minimalinvasive, katheterbasierte Verfahren in der Medizin sind offenen chirurgischen Maßnahmen hinsichtlich Krankenhausverweildauer, Intensität postoperativer Schmerzen und kosmetischen Ergebnissen überlegen. Aufgrund dieser ökonomischen, aber auch psychologischen Vorteile hat der Einsatz von Kathetersystemen seinen festen Platz in der Therapie von Herzerkrankungen gefunden. In der Behandlung von stenosierten Herzkranzgefäßen oder Aortenaneurysmata sind sie inzwischen bei definierten Indikationen gar Standard geworden. Ferner kommen Kathetersysteme routinemäBig bei der Therapie spezieller kongenitaler Herzvitien, wie dem Vorhofseptumdefekt, zum Einsatz. Der augenscheinliche Trend bewegt sich also auch in der Herzchirurgie zur Minimierung des Operationstraumas bei gleichzeitiger Reduktion der Inzidenz von perioperativer Morbidität und Letalität $[14,15,16,17]$.

Im Jahre 1992 hielten minimalinvasive Kathetersysteme Einzug in das Gebiet des Herzklappenersatzes. Anderson und Pavnik et al. [18] zeigten erstmals, dass es möglich ist, in einem nichtsternotomierten Tiermodell eine Herzklappe über einen transluminalen Zugang einzusetzen.

Bislang sind klinisch vor allem auf dem Gebiet des perkutanen Pulmonalklappenersatzes vielversprechende Ergebnisse erzielt worden. Nach einer Reihe erfolgreicher tierexperimenteller Studien bewiesen Bonhoeffer et al. im Jahr 2000 mit der ersten erfolgreichen Implantation bei einem zwölfjährigen Jungen die Praktikabilität dieses minimalinvasiven Verfahrens [19]. Zwei Jahre später berichtete wiederum die Gruppe um Bonhoeffer von sieben Kindern und einem erwachsenen Patienten mit signifikanter pulmonaler Regurgitation oder einer Obstruktion im RVOT, die transfemoral mit einer in einen
Platinumstent eingenähten biologischen Klappe versorgt worden sind [20]. Die auf diesem Gebiet führende Gruppe um Bonhoeffer präsentierte im Jahr 2005 weitere erfolgreiche Implantationen bei 59 Patienten. Es handelt sich hier in den meisten Fällen um Reinterventionen des RVOT bei juvenilen und erwachsenen Patienten mit Zustand nach operativer Korrektur einer Fehlbildung der Strukturen des RVOT (z.B. Fallot-Tetralogie) [21]. Mit dieser Vorzeigestudie stellte diese Arbeitsgruppe ihre weltweite Führung auf dem Gebiet der perkutanen Implantation von klappentragenden Stents wiederholt unter Beweis.

Entscheidend für den Erfolg dieser Methode ist die Patientenselektion für die perkutane Pulmonalklappenimplantation. Ein verkalkter Homograft bietet ein optimales Lager für die Implantation des klappentragenden Stents. In den meisten Fällen geht mit einem destruierten Homograft eine Kombination aus Stenose und Insuffizienz einher. Die stenotische Komponente ist für eine sichere Verankerung der neuen Klappe günstig.

Patienten mit einem nativen RVOT nach Fallot-Korrektur, bei denen z. B. lediglich eine Valvotomie durchgeführt wurde, sind hingegen keine geeigneten Kandidaten für eine perkutane Implantation, da sie keinen Verankerungspunkt auf Höhe des Pulmonalklappenannulus bieten. Bei Vorliegen eines dilatierten RVOT im Sinne eines Aneurysma mit Durchmessern über $24 \mathrm{~mm}$ ist eine perkutane Implantation bislang nicht durchzuführen.

Bonhoeffer wählt bei der perkutanen Implantation einen transfemoralen oder transjugulären Zugangsweg. In einigen wenigen Kliniken wurden in den letzten Monaten Pulmonalklappenstents transthorakal und -ventrikulär am schlagenden Herzen implantiert. Hierzu finden sich jedoch noch wenige wissenschaftliche Veröffentlichungen [22]. Berichte über erfolgreiche Implantationen in München und Freiburg waren lediglich bereits in Nachrichtensendungen zu hören und in der Tagespresse zu lesen. Dieser Zugangsweg erlaubt die Verwendung relativ großer Implantationsbestecke. Die Implantation ist so also unabhängig vom Durchmesser peripherer Gefäße und kann auch bei jüngeren und kleineren Patienten angewandt werden.

\section{Und was haben wir gemacht? („The Kiel Experience“)}

Der wahrscheinlich erste deutsche Herzchirurg, der sich Gedanken darüber machte, wie ein Herzklappenersatz über die Leiste durchführbar sein könnte, war Ende der 1990er Jahre der damals in Freiburg tätige Georg Lutter. Lutter war vom „Virus perkutaner Herzklappenersatz“ infiziert und leistete grundlegende Vorarbeiten für diese neuartige Methode. Man benötigt dazu eine faltbare Herzklappenprothese, die über einen geeigneten Katheter an die gewünschte Stelle im Gefäßsystem gebracht werden kann. Lutters Gruppe verwendete selbstexpandierende Stents, in welche eine porcine Aortenklappe eingenäht worden war. Ich selbst wurde zu diesem Zeitpunkt vom „Virus" als Doktorand in einem seiner weiteren Projekte infiziert, in welchem ich Großtierexperimente zur Evaluation der transmyokardialen Laserrevaskularisation durchführte. Es handelte sich um Langzeituntersuchungen an Schweinen. Es schien also nach umfangreichen In-vitro-Versuchen nahe liegend, dieses Tiermodell auch für den perkutanen Herzklappenersatz heranzuziehen. Die in stundenlanger Feinarbeit hergestellten klappentragenden Stents wurden über Bauchaorta oder Leistenarterie in die absteigende Aorta, die suprakoronare aufsteigende Aorta und in den linksventrikulären Ausflusstrakt infrakoronar implantiert. Problematisch waren damals der Durchmesser des Implantationskatheters, seine Steuerbarkeit, die Visualisierung des Implantationsvorgangs und die Verlegung der Koronarostien durch den Stent. Schließlich konnten 2002 die ersten Ergebnisse publiziert werden [23].

Gleichzeitig wurde in unserer Arbeitsgruppe am perkutanen Pulmonalklappenersatz gearbeitet. Forciert wurde diese Entwicklung durch die Zusammenarbeit mit dem damals in Jena tätigen Herzchirurgen Ulrich Stock. Seine Erfahrungen mit der Gewebezucht (tissue engineering) sollten mit dem minimalinvasiven Herzklappenersatz kombiniert werden. Theoretische Vorteile einer aus körpereigenen Zellen gezüchteten Herzklappenprothese sind deren lange Haltbarkeit und ihr Wachstumspotenzial. Beide Eigenschaften sind gerade bei der Behandlung von Kindern mit 
Z Herz- Thorax- Gefäßchir 2008 · 22:98-102

DOI 10.1007/s00398-008-0621-9

○) Springer Medizin Verlag 2008

\section{T. Attmann}

\section{Perkutaner Pulmonalklappen- ersatz: Warum macht man das? Wie macht man das? Und was haben wir gemacht? „The Kiel Experience"}

\section{Zusammenfassung}

Erst seit kurzem wird der katheterbasierte Herzklappenersatz klinisch angewandt. Bislang scheint insbesondere der perkutane Pulmonalklappenersatz zufriedenstellende Ergebnisse zu liefern. Im vorliegenden Artikel werden die Indikationen für den perkutanen Pulmonalklappenersatz sowie der Stand der klinischen Entwicklung dargestellt. Darüber hinaus beschreibt der Autor seine Erfahrungen mit der experimentellen Evaluation dieser innovativen Methode.

\section{Schlüsselwörter}

perkutan · Herzklappenersatz $\cdot$ Pulmonalklappe $\cdot$ Katheter

\section{Percutaneous pulmonary valve replacement: indications, stage of development and the Kiel experience}

\begin{abstract}
Transcatheter valve replacement has recently been introduced into clinical practice. To date, the largest human experience exists with percutaneous pulmonary valve implantation (PPVI) in patients with repaired congenital heart disease who require reintervention to the right ventricular outflow tract. In the present article the indications for PPVI and the stage of clinical development are delineated. Furthermore, a junior doctor gives a firsthand report of his experience with the experimental evaluation of this cutting-edge technique.
\end{abstract}

\section{Keywords}

percutaneous - valve replacement . pulmonary valve $\cdot$ catheter angeborenen Herzfehlern wünschenswert, für welche ja aus genannten Gründen die perkutane Implantation ebenfalls eine positive Entwicklung darstellen könnte.

Georg Lutter wechselte Anfang 2003 nach Kiel. Zwischenzeitlich war ich Arzt im Praktikum und verließ meine Freiburger Heimat, um in Kiel weiter in Lutters Arbeitsgruppe mit Fokus auf das DFG-geförderte Pulmonalklappenprojekt von Stock und Lutter zu forschen. Dies gestaltete sich zunächst äußerst schwierig, da es in Kiel keine adäquate Infrastruktur zur Durchführung von Großtierversuchen gab. Eine Abteilung für chirurgische Forschung mit Veterinärmedizinern und Tierpflegepersonal, wie wir sie aus Freiburg kannten, existierte nicht. Auch fehlte eine Röntgendurchleuchtungsanlage im experimentellen Operationssaal und der Tiertransport musste vom Projektleiter selbst durchgeführt werden. Glücklicherweise hatte dieser sich gerade einen Benz gekauft; Attmanns E-Klasse-Kombi diente also nun zum Schweinetransport.

Der Kinderkardiologe Grabitz stellte einen ausgedienten Katheterplatz zur Verfügung, Nitinol-Stents wurden mit porcinen Aortenklappen bestückt und über die Leiste implantiert. Nach wenigen Versuchen mussten wir feststellen, dass unsere Klappenstents erstens zu viel Platz im Implantationskatheter einnahmen, zweitens die Katheter insbesondere in Bezug auf ihre Steuerbarkeit nicht den notwendigen Anforderungen gerecht wurden und drittens das Schwein nicht das optimale Objekt für eine transfemorale Pulmonalklappenimplantation darstellte, da der Weg von der unteren Hohlvene in die Pulmonalarterie für einen Katheter doch zu verschlungen ist. Letztere Erkenntnis wurde uns von Alain Cribier, dem Pionier auf dem Gebiet des perkutanen Aortenklappenersatzes, in einem persönlichen Gespräch Anfang 2004 bestätigt. Zu allem Unglück verließ Herr Grabitz Kiel, sodass wir zu den drei genannten noch ein viertes Problem bekamen: wir hatten nun auch keinen Angiographiearbeitsplatz mehr.

\section{Wie wurden unsere vier Probleme nun angepackt?}

Um kleinere klappentragende Stents zu erhalten, entschieden wir, diese statt mit porcinen Aortenklappen mit bovinen Jugularvenen zu bestücken. Da eine Zusammenarbeit mit einem großen, herzklappenproduzierenden Unternehmen nicht möglich war, nahmen wir Kontakt zur Norddeutschen Fleischzentrale in Bad Bramstedt auf. Die Mitarbeiter sagten zu, regelmäßig Rinderhalsvenen zu liefern. Tatsächlich konnten wir wöchentlich eine große Menge an Rindergewebe abholen. Bedauerlicherweise enthielten diese Lieferungen meist Carotiden und keine Jugularvenen, und leider war dieser Unterschied auch nicht vermittelbar, weshalb diese Quelle versiegte. Erst als ein Doktorand eine kleine ostfriesische Schlachterei für unser Projekt auftat, war der Nachschub an Jugularvenen gesichert. Sie wurden präpariert und in die selbstexpandierenden Stents eingenäht (• Abb. 1). Diese Klappenstents konnten auf deutlich kleinere Durchmesser zusammengefaltet werden als die ursprünglichen, Aortenklappen enthaltenden Klappenstents.

Auch das zweite Problem, die mangelhafte Steuerbarkeit der eingesetzten Ka-

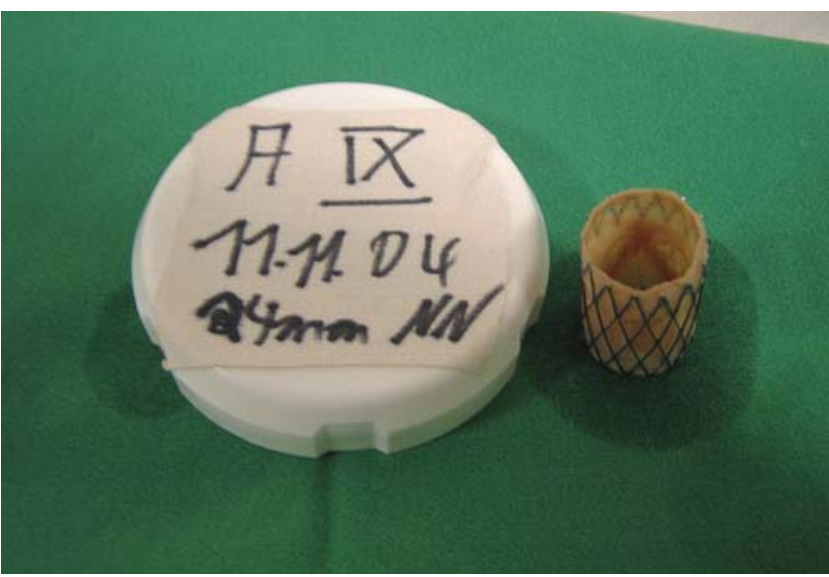

Abb. $1<$ Klappenstent Nummer 9: NitinolStent (Durchmesser $24 \mathrm{~mm}$ ) mit dem klappentragenden Segment einer bovinen Jugularvene 
theter, konnte gelöst werden: es wurde ein Katheter gefunden, der zur Implantation aortaler Endoprothesen dient. Nach Modifikationen an diesem Katheter konnten mit ihm die bovine Jugularvenen tragenden Stents implantiert werden.

All dies selbstredend erst nachdem der Antrag auf Versuche mit Schafen genehmigt und ein Schäfer gefunden wurde, bei dem Attmanns Wagen vorfahren und mit lebenden Tieren beladen werden konnte. Und natürlich ebenfalls erst nachdem ein neuer Angiographiearbeitsplatz bereit stand. Dieser wurde schließlich von Martin Heller, dem Direktor der Klinik für Diagnostische Radiologie, zur Verfügung gestellt. Die interventionellen Radiologen Stefan Müller-Hülsbeck und Thomas Jahnke halfen bei den Implantationen (- Abb. 2).

Am Schaf wurde nun ein transfemoraler Zugang realisiert. Bis dato kamen ausschließlich ballonexpandierbare Stents bei einer perkutanen Pulmonalklappenimplantation zum Einsatz. Selbstexpandierende Stents, welche meist aus Nitinol, einer Nickel-Titan-Legierung, gefertigt sind, besitzen theoretisch Vorteile bezüglich Hämodynamik - sowohl während des Implantationsvorgangs, als auch im Verlauf, gerade in den herznahen großen Gefäßen - und Langzeitfunktion der klappentragenden Stents selbst ( $\bullet$ Abb. $\mathbf{3}$ ).

An diese Machbarkeitsstudie [24] schloss sich eine Langzeituntersuchung an. Nach erfolgreicher Implantation des Klappenstents erwachten die Tiere aus der Narkose (• Abb. 2) und wurden zurück in den Stall gebracht, um dort die nächsten drei Monate zu verbringen; einen Stall zu besorgen, war übrigens das fünfte Problem. Mit dem Start der Langzeitstudie mussten wir deshalb warten, bis die Zusage für eine hartnäckig erbetene, kleine Ecke im Vorraum des Stalls des Instituts für Tierernährung kam (• Abb.4). Neben Herzsonographie und Computertomographie wurden in dieser Studie erstmals hämodynamische Untersuchungen während des Implantationsvorgangs durchgeführt. Mit dieser tierexperimentellen Studie konnte außerdem erstmals eine gute Langzeitfunktion von perkutan implantierten, selbstexpandierbaren Nitinol-Stents in Pulmonalisposition gezeigt werden [25].

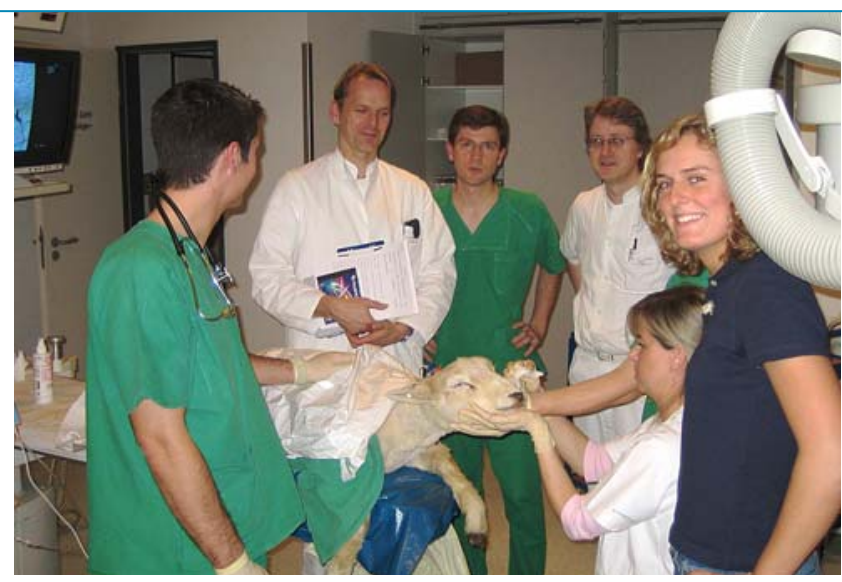

Abb. $2>$ Versuchstier kurz nach Extubation; Christian König, Stefan Müller-Hülsbeck, Tim Attmann, Thomas Jahnke, Beata Hoffmann und Kristin Rumberg (v.l.n.r.)

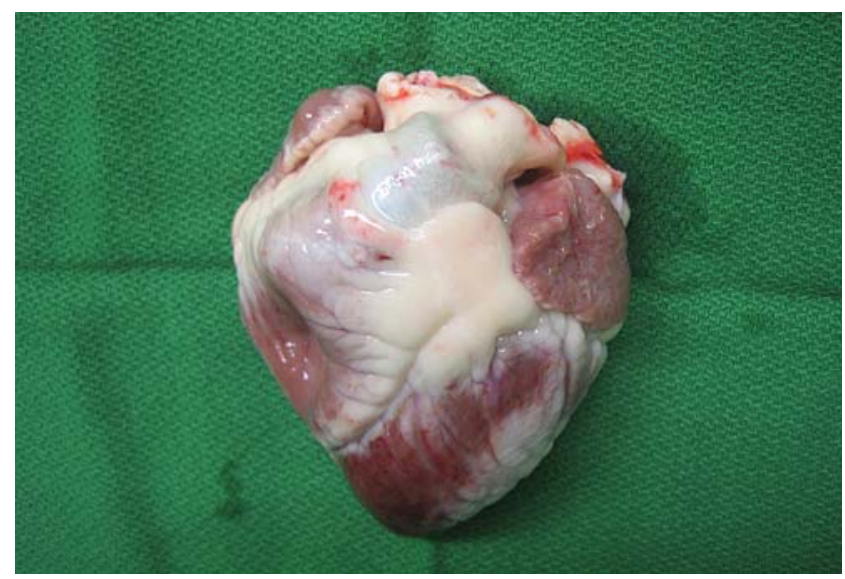

Abb. 3 - Schafherz nach Explantation; der klappentragende Stent zeichnet sich am rechtsventrikulären Ausflusstrakt ab

Abb.4 Die Ecke im Stall; der Projektleiter freut sich über die beiden ersten Tiere der Langzeituntersuchung mit Zustand nach perkutaner Implantation einer Pulmonalklappenprothese

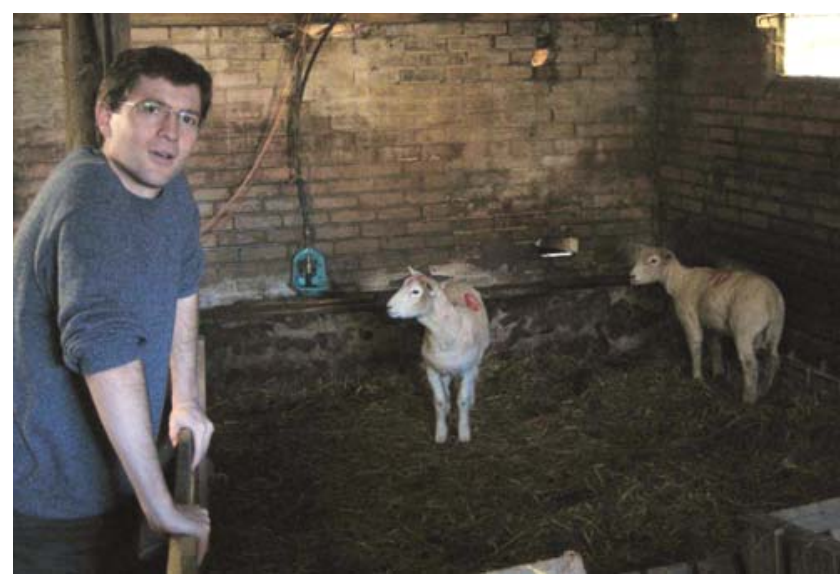

Parallel zu den In-vivo-Studien wurde an der Entwicklung neuer Kathetersysteme gearbeitet, die eine schonende und genauere Platzierung des klappentragenden Stents ermöglichen [26]. Getestet wurde in einem Herz-Gefäß-Modell aus Silikon.

Viele weitere Fragestellungen konnten nach Beseitigung der Hindernisse, die wir in Kiel antrafen, bearbeitet werden. Dazu gehörten Histologie und Verkalkungscharakteristika der Klappenstents sowie neue Materialien, aus denen Klappenstents gefertigt werden können, wie Polyurethan.

Unterdessen wurde ein klinikeigener Tiertransporter angeschafft, es wurde mit dem Bau eines Großtierlabors begonnen, welches für die Durchführung herzchirurgischer Operationen und angiographisch sein wird. Und es wird spezielle Intensivboxen und Stellplätze für die Versuchstiere geben. Künftige Forscher treffen in Kiel also gute Bedingungen an. Jedoch wird ihnen ggf. ein kleines bisschen Abenteuer fehlen. Aktuell ist in Kiel das Gewebezucht-Programm angelaufen. In Bioreaktoren besiedelt man biologische Herzklappengerüste mit Endothel- und glatten Muskelzellen, bevor sie via Leistenvene in Pulmonalisposition implantiert werden. gesteuerter Interventionen ausgestattet 


\section{Zusammenfassung}

Die katheterbasierte Implantation von Pulmonalklappen ist bei ausgewählten Patienten möglich und sicher durchführbar. Verkalkte Homografts sind am besten geeignet für eine perkutane Implantation. Bisher werden klinisch ballonexpandierbare Stents verwendet. In unseren tierexperimentellen Studien konnten wir zeigen, dass auch selbstexpandierende Stents geeignet sind. Über Langzeitfunktion und Haltbarkeit der perkutan implantierten Pulmonalklappen sind noch keine Aussagen möglich. Ungeachtet dessen können große Operationen mit Hilfe dieser neuen Technologie zumindest hinausgeschoben werden. Sie ist somit als Alternative bzw. zusätzliches Verfahren im Bereich der kongenitalen Herzchirurgie anzusehen. Mit zunehmender Erfahrung und sich einstellenden Langzeitergebnissen könnte die Indikation zum nichtchirurgischen Eingriff erweitert werden.

Individuelle, lebenslange Strategien für die Behandlung der Patienten mit kongenitalen Vitien des RVOT sind essenziell und von Kinderkardiologen, Kardiologen und Herzchirurgen gemeinsam auszuarbeiten.

Junge Kollegen sollten sich von kleineren Unwägbarkeiten keinesfalls abschrecken lassen, Forschung zu betreiben. Es macht Freude, mit Kollegen und Doktoranden zu diskutieren und sich gute Arbeitsbedingungen ggf. erst zu schaffen. Die Aussicht auf Reisen zu internationalen Kongressen, Veröffentlichungen und vielleicht sogar auf Preise motiviert, neben dem Klinikalltag auch mal ins Labor zu gehen.

\section{Literatur}

1. Blalock A, Hanlon $\mathrm{Cr}$ (1950) The surgical treatment of complete transposition of the aorta and the pulmonary artery. Surg Gynecol Obstet; 90:1-15

2. Blalock A, Kieffer Rf Jr (1950) Valvulotomy for the relief of congenital valvular pulmonic stenosis with intact ventricular septum; report of 19 operations by the Brock method. Ann Surg; 132:496-516

3. Rastelli GC, Ongley PA, McGoon DC (1969) Surgical repair of complete atrioventricular canal with anterior common leaflet undivided and unattached to ventricular septum. Mayo Clin Proc; 44:335-341

4. Yacoub MH, Radley-Smith R (1978) Anatomy of the coronary arteries in transposition of the great arteries and methods for their transfer in anatomical correction. Thorax; 33:418-424
5. Therrien J, Marx GR, Gatzoulis MA (2002) Late problems in tetralogy of Fallot-recognition, management, and prevention. Cardiol Clin; 20:395-404

6. Gatzoulis MA, Balaji S, Webber SA, Siu SC, Hokanson JS, Poile C, Rosenthal M, Nakazawa M, Moller JH, Gillette PC, Webb GD, Redington AN (2000) Risk factors for arrhythmia and sudden cardiac death late after repair of tetralogy of Fallot: a multicentre study. Lancet; 356:975-981

7. Therrien J, Siu SC, Harris L, Dore A, Niwa K, Janousek J, Williams WG, Webb G, Gatzoulis MA (2001) Impact of pulmonary valve replacement on arrhythmia propensity late after repair of tetralogy of Fallot. Circulation; 103:2489-2494

8. Gatzoulis MA, Elliott JT, Guru V, Siu SC, Warsi MA, Webb GD, Williams WG, Liu P, McLaughlin PR (2000) Right and left ventricular systolic function late after repair of tetralogy of Fallot. Am J Cardiol; 86:1352-1357

9. Helbing WA, Niezen RA, Le Cessie S, van der Geest RJ, Ottenkamp J, de Roos A (1996) Right ventricular diastolic function in children with pulmonary regurgitation after repair of tetralogy of Fallot: volumetric evaluation by magnetic resonance velocity mapping. J Am Coll Cardiol; 28:1827-1835

10. Gatzoulis MA, Till JA, Redington AN (1997) Depolarization-repolarization inhomogeneity after repair of tetralogy of Fallot. The substrate for malignant ventricular tachycardia? Circulation; 95:401-404

11. Therrien J, Siu SC, McLaughlin PR, Liu PP, Williams WG, Webb GD (2000) Pulmonary valve replacement in adults late after repair of tetralogy of fallot: are we operating too late? J Am Coll Cardiol; 36:1670-1675

12. Wells WJ, Arroyo Jr H, Bremner RM, Wood J, Starnes VA (2002) Homograft conduit failure in infants is not due to somatic outgrowth. J Thorac Cardiovasc Surg; 124:88-96

13. Kanter KR, Budde JM, Parks WJ, Tam VK, Sharma S, Williams WH, Fyfe DA (2002) One hundred pulmonary valve replacements in children after relief of right ventricular outflow tract obstruction. Ann Thorac Surg; 73:1801-1806

14. Byrne JG, Hsin MK, Adams DH et al (2000) Minimally invasive direct access heart valve surgery. J Card Surg; 15:21-34

15. Grossi EA, Galloway AC, Ribakove GH et al (2001) Impact of minimally invasive valvular heart surgery: a case-controll study. Ann Thorac Surg; 71:807-810

\section{Korrespondierender Autor}

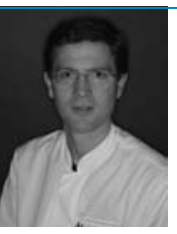

Dr. med. Tim Attmann

Klinik für Herz- und Gefäßchirurgie, Universitätsklinikum Schleswig-Holstein Campus Kiel

Arnold-Heller-Straße 7, 24105 Kiel, BRD

E-Mail: timattmann@web.de

Tim Attmann, Jahrgang 1975, studierte Medizin an der Albert-Ludwigs-Universität Freiburg i. Br. und promovierte über „Evaluation transgener und transmuraler Myokardrevaskularisation am chronisch ischämischen Modell anhand objektiver Parameter der regionalen und globalen Funktion" in der Abteilung für Herz- und Gefäßchirurgie am Universitätsklinikum Freiburg. Im Jahr 2003 wechselte er an die Klinik für Herz- und Gefäßchirurgie am Universitätsklinikum Schleswig-Holstein, Campus Kiel. Er forschte dort auf dem Gebiet des perkutanen Herzklappenersatzes und entwickelte neuartige Klappenstents und Kathetersysteme. Darüber hinaus hat Tim Attmann den Aufbau des Großtierlabors vorangetrieben. Seit 2008 ist er in der Klinik für Herz-, Kinderherz- und Gefäßchirurgie am Universitätsklinikum Gießen tätig.

Interessenkonflikt. Der korrespondierende Autor gibt an, dass kein Interessenkonflikt besteht. 